\title{
RESISTENCIA A CIZALLADURA EN JUNTAS DE Eucalyptus grandis Y Pinus elliottii DEL NORESTE DE ARGENTINA ENCOLADAS CON ADHESIVO ESTRUCTURAL
}

\author{
Juan Carlos Piter*, Carlos Fabio Calvo**, Ariel Anselmo Gonzalez***, \\ María Alexandra Sosa Zitto**, Pablo Marcelo Stefani ****, \\ Eduardo Antonio Torrán**, Dora Inés Villalba** \\ *Ing., Dr., Facultad Regional C. del Uruguay, UTN - piterj@frcu.utn.edu.ar- \\ **Ing., Facultad Regional C. del Uruguay, UTN - calvof@frcu.utn.edu.ar - sosazittoa@frcu.utn.edu.ar - \\ etorran@frcu.utn.edu.ar - villalbad@frcu.utn.edu.ar \\ ***Mag. Ing., Facultad Regional Santa Fé y Facultad Regional C. del Uruguay, UTN - aagonzal@frsf.utn.edu.ar \\ ****Ing., Dr., Facultad Regional C. del Uruguay, UTN, Facultad de Ingeniería, UNMP-CONICET - pmstefan@fi.mdp.edu.ar \\ Recebido para publicação: 03/07/2006 - Aceito para publicação: 30/09/2006
}

\begin{abstract}
Resumo
En esta publicación se presentan los resultados de un trabajo que forma parte de un proyecto de investigación orientado a estudiar el comportamiento de la madera de Eucalyptus grandis de Argentina encolada con adhesivos de uso estructural. Se diseñó un programa experimental para comparar el desempeño de esta especie con el Pinus elliottii, tomado como referencia. Las uniones de ambas especies se sometieron al esfuerzo de cizalladura por tracción luego de realizar los 3 tratamientos previstos en la norma IRAM 45055 (Instituto Argentino de Normalización, 2002b) para adhesivos estructurales aptos para clima interior. Los resultados obtenidos muestran un comportamiento similar para ambas especies. La aplicación de distintos niveles de presión no produjo diferencias de importancia en el desempeño de las uniones. Las probetas de Eucalyptus grandis preparadas en clima normalizado, con temperatura de $20( \pm 2){ }^{\circ} \mathrm{C}$ y humedad relativa ambiente de 65 $( \pm 5) \%$, se comportaron de manera similar a otras de la misma especie elaboradas en un ambiente con mayor temperatura $\left(23^{\circ} \mathrm{C}\right)$ y humedad relativa ambiente $(84 \%)$.

Palabras-clave: Eucalyptus grandis; Pinus elliottii; madera laminada encolada; cizalladura por tracción longitudinal.
\end{abstract}

\begin{abstract}
Shear strength in joints of Eucalyptus grandis and Pinus elliottii glued with structural adhesive. The present paper reports the results of an investigation regarding the behaviour of Argentinean Eucalyptus grandis glued with adhesives for load-bearing timber structures. An empirical research project was designed for comparing the performance of this species with that of Pinus elliottii, which was considered as a reference species. Joints were tested in longitudinal tensile shear after applying the 3 cycles corresponding to adhesives for indoor climate according to the standard IRAM 45055 (Instituto Argentino de Normalización, 2002b). Results showed a similar behaviour for both species. The performance of joints subjected to different pressure levels did not exhibit important differences. The specimens of Eucalyptus grandis prepared in normal climate, at $20( \pm 2){ }^{\circ} \mathrm{C}$ temperature and 65 $( \pm 5) \%$ relative humidity, showed a similar behaviour to those of the same species prepared in a climate with higher temperature $\left(23^{\circ} \mathrm{C}\right)$ and relative humidity $(84 \%)$.

Keywords: Eucalyptus grandis; Pinus elliottii; glued laminated timber; longitudinal tensile shear.
\end{abstract}

\section{INTRODUCCIÓN}

La madera laminada encolada es un material que ofrece excelentes propiedades para uso estructural y que amplía las posibilidades de utilización de la madera aserrada, pudiendo incluso competir ventajosamente en numerosas aplicaciones con otros materiales tradicionales. El desarrollo de numerosas investigaciones y la implementación de normas referidas a los requisitos que se deben cumplir en la 
fabricación, así como la adopción de clases resistentes, convierten a la madera laminada encolada en un producto con adecuada confiabilidad (COLLING, 1995a). Considerando que para el comportamiento del elemento estructural laminado son tan importantes las propiedades resistentes y de rigidez de las láminas como la calidad de la adhesión entre ellas y en particular de las uniones dentadas (COLLING; EHLBECK,1992), la realización de numerosos ensayos se ha complementado con el desarrollo de modelos que permiten simular su comportamiento mecánico con el apoyo de una computadora (COLLING, 1995b). Por su parte, los adhesivos para uso estructural, tanto para uso interior como exterior, deben garantizar un adecuado desempeño de las uniones durante la vida útil de la estructura (RADOVÍC, 1995; RAKNES, 1995).

La normativa disponible en el plano internacional, producida por países con una amplia experiencia en el uso estructural de la madera, permite calcular las propiedades de resistencia y rigidez de elementos laminados encolados construidos con una o más calidades de láminas (AMERICAN SOCIETY FOR TESTING AND MATERIALS, 1999; EUROPÄISCHES KOMITEE FÜR NORMUNG, 1999). En Europa, los requisitos que deben cumplir los adhesivos, y su clasificación, se encuentran establecidos en la norma EN 301 (EUROPÄISCHES KOMITEE FÜR NORMUNG, 2001).

Existe mayor experiencia acumulada con madera laminada encolada de especies de coníferas que de frondosas, lo cual en algunos casos se ve reflejado en las normas que limitan su campo de aplicación a las primeras (EUROPÄISCHES KOMITEE FÜR NORMUNG, 1999). Las fuerzas de unión entre la madera y el adhesivo se producen a través de adhesión química y mecánica. La adhesión química se basa en fuerzas intermoleculares que actúan en la superficie de contacto entre el adhesivo y la madera. La adhesión mecánica se materializa a través de un anclaje del adhesivo en la estructura interna de la madera (RAKNES, 1995; RADOVÍC, 1995). La microestructura anatómica de las especies frondosas es más compleja que la de las coníferas (HOFFMEYER, 1995) y sobre el comportamiento de este tipo de uniones tiene influencia la presión empleada en el prensado, que en general debe ser mayor en las frondosas que en las coníferas (RADOVÍC, 1995). GLOS y LEDERER (2000) reportaron problemas de adhesión en uniones para uso estructural originados en la estructura interna de algunas especies frondosas, como es el caso del Roble europeo (Quercus robur L).

La redacción de los proyectos de normas IRAM 9660-1/2, 9661 y 9662-1/2/3, actualmente en proceso de aprobación, representa un avance en la obtención de un sistema de calidad para la madera laminada encolada en Argentina. El mismo contempla la utilización de 3 especies forestadas en el país, cuales son la Araucaria angustifolia cultivada en la Provincia de Misiones, el Pinus elliottii y el Pinus taeda cultivados en la Provincia de Misiones y el norte de Corrientes y el Eucalyptus grandis cultivado en las provincias de Entre Ríos, Corrientes y Misiones. Existe abundante experiencia que prueba la aptitud de las dos primeras especies para ser encoladas con adhesivos de uso estructural, pero es menor la información disponible sobre ese aspecto acerca del Eucalyptus grandis. A su vez, esta última especie, cuyo manejo forestal, secado técnico y utilización se han incrementado últimamente, representa un recurso de importancia para el país (INTA, 1995). Investigaciones previas (PITER et al., 2004) han reportado que el Eucalyptus grandis cultivado en la Mesopotamia de Argentina presenta una densidad aparente sensiblemente menor a la misma especie cultivada en Australia, y que la relación entre su módulo de elasticidad y su densidad es más similar a la presente en las coníferas que a la que exhiben las especies frondosas conforme al sistema internacional de clases resistentes para madera aserrada adoptado por la la norma europea EN 338 (EUROPÄISCHES KOMITEE FÜR NORMUNG, 2003).

El objetivo de este trabajo es presentar y discutir los resultados de una investigación realizada con probetas de Eucalyptus grandis y Pinus elliottii del Noreste de Argentina, encoladas con un adhesivo estructural certificado para uso interior y sometidas a esfuerzos de cizalladura, y también, a través de la ejecución de los ensayos en las mismas condiciones para ambas especies, comparar la aptitud para el encolado del Eucalytus grandis con la del Pinus elliottii.

\section{MATERIAL Y MÉTODOS}

La madera de Eucalyptus grandis fue seleccionada al azar de una forestación de 26 años de edad, sembrada con semilla de origen sudafricano Transvaal, cultivada en Virasoro, Provincia de Corrientes. Esta región de cultivo es una de las más importantes de Argentina para la especie (INTA, 1995). La madera de Pinus elliottii fue obtenida con igual criterio y de la misma región, de una forestación de 18 
años de edad sembrada con semilla de origen Saint Joint. Luego del secado, el material se acondicionó en clima normalizado, con $20( \pm 2){ }^{\circ} \mathrm{C}$ de temperatura y $65( \pm 5) \%$ de humedad relativa ambiente. El contenido de humedad de la madera luego de su estacionamiento en el clima normalizado fue determinado conforme a los lineamientos de la norma ISO 3130 (INTERNATIONAL ORGANIZATION FOR STANDARDIZATION, 1975a) y la densidad aparente de acuerdo con la ISO 3131 (INTERNATIONAL ORGANIZATION FOR STANDARDIZATION, 1975b).

Con la madera estabilizada se prepararon 5 bloques de paneles destinados a la producción de las probetas, siguiendo los procedimientos indicados en la norma IRAM 45054-1 (INSTITUTO ARGENTINO DE NORMALIZACIÓN, 2002a) para películas delgadas de adhesivo, la que se encuentra en línea con la norma europea EN 302-1 (EUROPÄISCHES KOMITEE FÜR NORMUNG, 2004). Cada bloque reunió las características que se ilustran en la tabla 1. La cantidad de probetas respondió al propósito de contar con un mínimo de 10 resultados válidos al final del proceso para cada bloque y para cada uno de los 3 tratamientos previstos en la norma IRAM 45055 (INSTITUTO ARGENTINO DE NORMALIZACIÓN, 2002b) para adhesivos de uso en clima interior, la que adopta criterios similares a los estipulados en la EN 301 (EUROPÄISCHES KOMITEE FÜR NORMUNG, 2001). Como adhesivo se empleó una resina sintética (urea-formol-melamina) que cumple con las especificaciones de la norma IRAM 45055 citada anteriormente. Siguiendo las instrucciones del fabricante se efectuó la preparación de la mezcla y se esparcieron $250 \mathrm{~g} / \mathrm{m}^{2}$ sobre una de las caras a unir, empleando un pincel, en iguales condiciones para ambas especies.

Tabla 1. Material empleado en los ensayos.

Table 1. Test samples.

\begin{tabular}{lccc}
\hline Bloques & Especie & $\begin{array}{c}\text { Presión } \\
\left(\mathbf{N} / \mathbf{m m}^{\mathbf{2}}\right)\end{array}$ & $\begin{array}{c}\text { Cantidad de } \\
\text { probetas }\end{array}$ \\
\hline E 0,4 & Eucalyptus grandis & 0,4 & 36 \\
E 0,8 & Eucalyptus grandis & 0,8 & 48 \\
P 0,4 & Pinus elliottii & 0,4 & 48 \\
P 0,8 & Pinus elliottii & 0,8 & 48 \\
E $0,4 b$ & Eucalyptus grandis & 0,4 & 48 \\
\hline
\end{tabular}

Teniendo en cuenta que el fabricante recomienda un valor mínimo de $0,4 \mathrm{~N} / \mathrm{mm}^{2}$ para la presión de prensado, y con el propósito de estudiar la influencia de esta variable, en los bloques $E 0,4$ y $P 0,4$ se aplicó ese valor mínimo recomendado y en otro bloque de cada especie se empleó una presión de 0,8 $\mathrm{N} / \mathrm{mm}^{2}(E \quad 0,8$ y $P$ 0,8).

Los bloques $E 0,4, E 0,8, P 0,4$ y $P 0,8$ se prepararon y prensaron en clima normalizado ( $\mathrm{T}=20$ $\pm 2{ }^{\circ} \mathrm{C}$ y HRA $=65 \pm 5 \%$ ). Para evaluar la influencia del clima imperante en este proceso, se elaboró un bloque adicional con madera de Eucalyptus grandis sometido a una presión de $0,4 \mathrm{~N} / \mathrm{mm}^{2}$ (bloque $E$ $0,4 b)$, con iguales características que el primer bloque $(E 0,4)$ pero preparado en clima interior no normalizado $\left(\mathrm{T}=23^{\circ} \mathrm{C}\right.$ y HRA $\left.=84 \%\right)$.

Luego de un tiempo mínimo de prensado de 24 horas en las condiciones ya descriptas, los 5 bloques se mantuvieron en atmósfera normalizada durante al menos 7 días. Con posterioridad se cortaron y prepararon las probetas (ver Figura 1), procediéndose a realizar los 3 tratamientos que contempla la norma IRAM 45055 (INSTITUTO ARGENTINO DE NORMALIZACIÓN, 2002b) para adhesivos aptos para clima interior: Tratamiento $A 1$ ) estacionamiento de las probetas durante 7 días en clima normalizado, ensayándolas luego secas; Tratamiento $A 2$ ) estacionamiento de las probetas durante 7 días en clima normalizado, luego 4 días sumergidas en agua a $15( \pm 5)^{\circ} \mathrm{C}$, ensayándolas luego húmedas; Tratamiento A3) estacionamiento de las probetas durante 7 días en clima normalizado, luego 4 días sumergidas en agua a $15( \pm 5)^{\circ} \mathrm{C}$, luego 7 días en clima normalizado, ensayándolas luego secas.

Después de finalizados los tratamientos, los ensayos de cizalladura por tracción se llevaron a cabo en una máquina de ensayos universales Shimadzu UH $1000 \mathrm{kN}$, con capacidad para aplicar la carga longitudinal $(F)$ desplazando el cabezal con velocidad constante y adecuada precisión (ver Figura 1). Las determinaciones estadísticas se realizaron para cada tratamiento y cada bloque con el número de ensayos válidos $(n)$. Con los resultados de la fuerza de rotura por cizalladura $\left(F_{v}\right)$ se calculó la media aritmética $\left(F_{v, \text { med }}\right)$, como medida de tendencia central, y se registró el valor mínimo $\left(F_{v, \text { min }}\right)$ para evaluar el rango 
inferior de las variaciones, el cual es un valor estipulado en la normativa nacional e internacional (INSTITUTO ARGENTINO DE NORMALIZACIÓN, 2002b; EUROPÄISCHES KOMITEE FÜR NORMUNG, 2001). Para mensurar la dispersión de los valores de $F_{v}$ se calculó el coeficiente de variación $(\mathrm{COV})$. Como variable complementaria a la fuerza de rotura, orientada a evaluar la calidad de las juntas, se determinó el porcentaje de fallo por madera y se calculó su valor medio $\left(R M_{\text {med }}\right)$ expresado como porcentaje, conforme lo prescripto en la norma IRAM 45054-1 (INSTITUTO ARGENTINO DE NORMALIZACIÓN, 2002a).

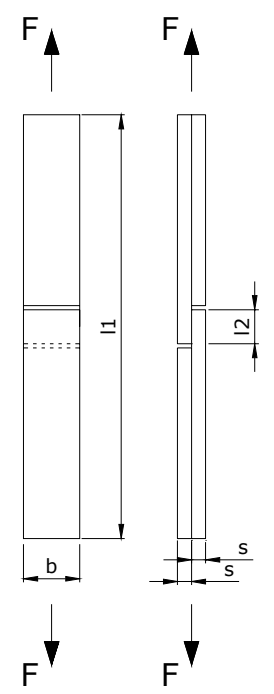

Figura 1. Probeta. $\mathrm{b}=(20,0 \pm 0,1) \mathrm{mm}, 11=(150 \pm 5) \mathrm{mm}, 12=(10 \pm 0,1) \mathrm{mm}, \mathrm{s}=(5,0 \pm 0,1) \mathrm{mm}$.

Figure 1. Test specimen. $\mathrm{b}=(20,0 \pm 0,1) \mathrm{mm}, 11=(150 \pm 5) \mathrm{mm}, 12=(10 \pm 0,1) \mathrm{mm}, \mathrm{s}=(5,0 \pm 0,1) \mathrm{mm}$.

\section{RESULTADOS Y DISCUSIÓN}

El valor medio de la densidad aparente para el Eucalyptus grandis fue de $564 \mathrm{~kg} / \mathrm{m}^{3}$, con un contenido de humedad de 11,4 \%. En el Pinus elliottii el valor medio de la densidad aparente fue de 534 $\mathrm{kg} / \mathrm{m}^{3}$, con un contenido de humedad de $11,9 \%$. Se han reportado valores de la densidad aparente para las mismas especies y procedencias obtenidos en investigaciones previas. Piter (2005) informó valores característicos de $420 \mathrm{~kg} / \mathrm{m}^{3}$ y $430 \mathrm{~kg} / \mathrm{m}^{3}$ para la madera de Pinus elliottii y de Eucalyptus grandis, respectivamente, con un $12 \%$ de contenido humedad, mientras que Piter et al. (2004) reportaron un valor característico de $430 \mathrm{~kg} / \mathrm{m}^{3}$ y un valor medio de $560 \mathrm{~kg} / \mathrm{m}^{3}$ para el Eucalyptus grandis con un $12 \%$ de contenido de humedad. Este último valor medio es prácticamente coincidente con el encontrado para la misma especie en la presente investigación. La tabla 2 exhibe los principales resultados obtenidos para los 3 tratamientos previstos con los bloques $E 0,4, E 0,8, P 0,4$ y $P 0,8$, habiéndose alcanzado en todos los casos la cantidad mínima de 10 ensayos válidos prevista en la norma IRAM 45054-1 (INSTITUTO ARGENTINO DE NORMALIZACIÓN, 2002a).

Un análisis del comportamiento de ambas especies muestra que luego del tratamiento $A 1$ los valores medios de las fuerzas de rotura para la presión de $0,4 \mathrm{~N} / \mathrm{mm}^{2}$ son levemente superiores en el Eucalyptus grandis $\left(F_{v, \text { med }}=1,4 \mathrm{kN}\right.$ en $\left.E 0,4\right)$ que en el Pinus elliottii $\left(F_{v, \text { med }}=1,3 \mathrm{kN}\right.$ en $\left.P 0,4\right)$. Esta diferencia se acentúa para la presión de $0,8 \mathrm{~N} / \mathrm{mm}^{2}$, con $F_{v, \text { med }}=1,9 \mathrm{kN}$ en $E 0,8$ y $F_{v, \text { med }}=1,4 \mathrm{kN}$ en $P$ 0,8. Los valores mínimos $\left(F_{v, \text { min }}\right)$ exhiben pequeñas diferencias, siendo superiores en el Eucalyptus grandis para la menor presión y en el Pinus elliottii para la mayor. Por su parte, esta última especie muestra una dispersión sensiblemente mayor, con niveles del $C O V$ de $32 \%$ y $37 \%$ para $P 0,4$ y $P 0,8$, respectivamente, mientras que el Eucalyptus grandis exhibe un $C O V$ igual a $24 \%$ para $E 0,4$ y $E 0,8$. El porcentaje medio de fallo por madera $\left(R M_{\text {med }}\right)$ es igual en $E 0,4$ que en $P 0,4(80 \%)$ pero sensiblemente mayor en $P 0,8(90 \%)$ que en $E 0,8(60 \%)$, coincidiendo en este último caso con el mayor nivel obtenido para $F_{v, \text { med }}$. 
Tabla 2. Resultados de los ensayos para los bloques E 0,4, E 0,8, P 0,4 y P 0,8.

Table 2. Results for the blocks E 0,4 , E 0,8, P 0,4 y P 0,8 .

\begin{tabular}{lcccccc}
\hline Tratamiento & Bloque & $\boldsymbol{n}$ & $\boldsymbol{F}_{\boldsymbol{v}, \text { med }}(\mathbf{k N})$ & $\boldsymbol{F}_{\boldsymbol{v}, \boldsymbol{m i n}}(\mathbf{k N})$ & $\boldsymbol{C O} \boldsymbol{V}(\mathbf{\%})$ & $\boldsymbol{R M}_{\boldsymbol{m e d}}(\mathbf{\%})$ \\
\hline$A 1$ & $E 0,4$ & 10 & 1,4 & 1,0 & 24 & 80 \\
& $E 0,8$ & 13 & 1,9 & 0,9 & 24 & 60 \\
& $P 0,4$ & 11 & 1,3 & 0,8 & 32 & 80 \\
& $P 0,8$ & 10 & 1,4 & 1,0 & 37 & 90 \\
\hline$A 2$ & $E 0,4$ & 12 & 1,0 & 0,4 & 45 & 50 \\
& $E 0,8$ & 14 & 1,2 & 0,5 & 41 & 70 \\
& $P 0,4$ & 16 & 1,1 & 0,5 & 36 & 40 \\
& $P 0,8$ & 13 & 1,3 & 0,6 & 29 & 70 \\
\hline$A 3$ & $E 0,4$ & 11 & 1,5 & 0,6 & 48 & 80 \\
& $E 0,8$ & 13 & 1,5 & 0,6 & 37 & 40 \\
& $P 0,4$ & 12 & 1,5 & 0,8 & 32 & 80 \\
& $P 0,8$ & 11 & 1,4 & 0,8 & 41 & 80 \\
\hline
\end{tabular}

$n \overline{\text { número de ensayos válidos; } F_{v, \text { med }}} \overline{\text { y } F_{v, \text { min }} \text { : Valores medio y mínimo de la fuerza de rotura por cizalladura, respectivamente; }}$ $C O V$ : coeficiente de variación de la fuerza de rotura por cizalladura; $R M_{\text {med }}$ : valor medio del porcentaje de fallo por madera.

Para el tratamiento $A 2$, contrariamente al caso anterior, $F_{v, \text { med }}$ es levemente mayor en el Pinus elliottii que en el Eucalyptus grandis para ambas presiones, y $F_{v, \min }$ exhibe valores muy similares para las dos especies. También contrariamente al tratamiento anterior, la dispersión es sensiblemente mayor en el Eucalyptus grandis que en el Pinus elliottii. Por su parte, $R M_{m e d}$ es similar para los dos tipos de madera.

Luego del tratamiento $A 3$ se encuentran valores de $F_{v \text {,med }}$ prácticamente iguales para los 4 bloques, comprendidos entre $1,4 \mathrm{kN}$ y $1,5 \mathrm{kN}$, con una dispersión significativamente mayor en el Eucalyptus grandis para la menor presión y levemente menor para la mayor presión. Curiosamente, $F_{v, \text { min }}$ alcanza valores iguales para las probetas de Eucalyptus grandis sometidas a los 2 niveles de presión $(0,6$ $\mathrm{kN})$ y lo mismo sucede para las probetas de Pinus elliotiti $(0,8 \mathrm{kN})$. $R M_{\text {med }}$ es igual a $80 \%$ en $E 0,4, P 0,4$ y $P 0,8$, alcanzando $40 \%$ en $E 0,8$.

Cuando el ensayo de cizalladura por tracción se realiza con el objetivo de certificar la aptitud de un adhesivo estructural nuevo, para lo cual se deben utilizar probetas de la especie de referencia internacional (Fagus sylvatica), los valores mínimos de la fuerza de rotura $\left(F_{v, \text { min }}\right)$ estipulados en la normativa de Europa y de Argentina son $2,0 \mathrm{kN}, 1,2 \mathrm{kN}$ y $1,6 \mathrm{kN}$ para los tratamientos $A 1, A 2$ y $A 3$, respectivamente (EUROPÄISCHES KOMITEE FÜR NORMUNG, 2001; INSTITUTO ARGENTINO DE NORMALIZACIÓN, 2002b). En el presente trabajo esos valores no son alcanzados y la rotura se produjo en su mayor parte por cizalladura en la madera y no por defectos de adhesión, lo cual se manifiesta en el elevado nivel encontrado para $R M_{\text {med }}$ (ver Tabla 2). A su vez, los valores encontrados en este trabajo para $F_{v, \text { min }}$ son coherentes con la resistencia a la cizalladura que presenta la madera de Eucalyptus grandis y de Pinus elliottii, con las cuales fueron construidas las probetas ensayadas (PITER et al., 2004; PITER, 2005; EUROPÄISCHES KOMITEE FÜR NORMUNG, 2003).

Los resultados muestran que, para propósitos prácticos, puede considerarse que las juntas de madera de Eucalyptus grandis exhiben un comportamiento similar que las de Pinus elliottii cuando se las somete al ensayo de cizalladura por tracción, luego de ejecutar los 3 tratamientos antes detallados.

El análisis de los datos presentados en la tabla 2 puede realizarse también para cada especie en función de la presión aplicada. Las probetas sometidas a la mayor presión exhiben valores más altos de $F_{v, \text { med }}$ en ambas especies para los dos primeros tratamientos, pero no así para el tercero. No obstante, Las diferencias son leves, con excepción de la registrada para el Eucalyptus grandis en el tratamiento Al (1,9 $\mathrm{kN}$ en $E 0,8$ y $1,4 \mathrm{kN}$ en $E 0,4)$. La dispersión tiende a disminuir con el aumento de presión en el Eucalyptus grandis, con excepción del tratamiento $A 1$, en el cual el $C O V$ se mantiene invariable. Por el contrario, en el Pinus elliottii solamente se observa en el tratamiento $A 2$ una menor dispersión para una mayor presión, ya que para $A 1$ y $A 3$ el nivel del $C O V$ es mayor en $P 0,8$ que en $P 0,4$. Tampoco se aprecia una relación clara entre la presión y el porcentaje de rotura por madera. El Pinus elliottii exhibe un valor de $R M_{\text {med }}$ que crece significativamente con el nivel de presión para $A 2$ (40\% para $P 0,4$ y $70 \%$ para $P$ 0,8 ), pero esa diferencia se atenúa en $A 1$ y se anula en $A 3$. También en el Eucalyptus grandis, $R M_{m e d}$ es significativamente más alto para la mayor presión en $A 2(50 \%$ para $E 0,4$ y $70 \%$ para $E 0,8)$, pero sucede 
lo opuesto para $A 1$ y $A 3$.

Puede afirmarse, en consecuencia, que un aumento de la presión de prensado desde $0,4 \mathrm{~N} / \mathrm{mm}^{2}$ hasta $0,8 \mathrm{~N} / \mathrm{mm}^{2}$ no produce cambios significativos en el comportamiento de las uniones de ambas especies, y en este caso no se encuentra en línea con la recomendación de aplicar una mayor presión en las especies frondosas que en las coníferas (RADOVÍC, 1995).

Tabla 3. Resultados de los ensayos para el bloque E $0,4 \mathrm{~b}$.

Table 3. Results for the block E 0,4b.

\begin{tabular}{lcccccc}
\hline Tratamiento & Bloque & $\boldsymbol{n}$ & $\boldsymbol{F}_{\boldsymbol{v}, \text { med }}(\mathbf{k N})$ & $\boldsymbol{F}_{\boldsymbol{v}, \boldsymbol{m i n}}(\mathbf{k N})$ & $\boldsymbol{C O} \boldsymbol{V}(\%)$ & $\boldsymbol{R} \boldsymbol{M}_{\boldsymbol{m e d}}(\mathbf{\%})$ \\
\hline$A 1$ & $E 0,4 b$ & $15(10)$ & $1,3(1,4)$ & $0,7(1,0)$ & $32(24)$ & $90(80)$ \\
$A 2$ & $E 0,4 b$ & $14(12)$ & $1,1(1,0)$ & $0,5(0,4)$ & $44(45)$ & $90(50)$ \\
$A 3$ & $E 0,4 b$ & $16(11)$ & $1,4(1,5)$ & $0,5(0,6)$ & $35(48)$ & $70(80)$ \\
\hline
\end{tabular}

$n$ : número de ensayos válidos; $F_{v, \text { med }}$ y $F_{v, \text { min }}$ : Valores medio y mínimo de la fuerza de rotura por cizalladura, respectivamente; $C O V$ : coeficiente de variación de la fuerza de rotura por cizalladura; $R M_{\text {med }}$ : valor medio del porcentaje de fallo por madera.

Los resultados correspondientes al bloque adicional $(E 0,4 b)$ se encuentran en la tabla 3, donde se presentan también los de su similar original $(E 0,4)$, entre paréntesis, para facilitar la comparación de ambos. Como puede observarse, los valores de $F_{v, \text { med }}$ son prácticamente equivalentes para los dos bloques. La dispersión, medida a través del valor del $C O V$, es sustancialmente mayor en $E 0,4 b$ para $A 1$, prácticamente igual en ambos bloques para $A 2$ y sustancialmente menor en $E 0,4 b$ para $A 3$. $R M_{\text {med }}$ acusa un valor levemente mayor en $E 0,4 b$ para $A 1$, significativamente más alto para $A 2$ y algo menor para $A 3$.

Los resultados muestran que la elaboración del Bloque $E$ 0,4 en clima normalizado no produjo cambios de consideración en su comportamiento mecánico respecto del Bloque $E 0,4 b$ ejecutado fuera de esa atmósfera. Los valores obtenidos son congruentes con las recomendaciones del fabricante del adhesivo, que prescribe una temperatura mayor o igual a $18{ }^{\circ} \mathrm{C}$ en el momento del encolado pero sin hacer referencia a la humedad relativa ambiente. En ese aspecto, las normas europeas estipulan una temperatura mayor o igual a $15{ }^{\circ} \mathrm{C}$ y una humedad relativa ambiente comprendida entre $40 \quad \%$ y $75 \%$ (EUROPÄISCHES KOMITEE FÜR NORMUNG, 1996). No obstante, la preparación del Bloque E 0,4b en un ambiente con una humedad relativa ambiente de $84 \%$ no evidencia consecuencias de importancia en su comportamiento para propósitos prácticos.

\section{CONCLUSIONES}

Fue posible realizar ensayos de cizalladura por tracción en probetas de Eucalyptus grandis y Pinus elliottii, cultivados en el Noreste de Argentina, encolados con adhesivo estructural certificado para uso interior. Los resultados, obtenidos luego de someter las probetas a los tratamientos previstos en la norma IRAM 45054-1 (INSTITUTO ARGENTINO DE NORMALIZACIÓN, 2002a), confirman un comportamiento similar entre el Eucalyptus grandis y el Pinus elliottii, encontrándose un mayor porcentaje de rotura por cizalladura en la madera que por defectos de adhesión. No se observaron variaciones de importancia en el desempeño de las uniones de ambas especies cuando las probetas fueron prensadas con la presión mínima recomendada por el fabricante $\left(0,4 \mathrm{~N} / \mathrm{mm}^{2}\right)$ o con una superior $(0,8$ $\mathrm{N} / \mathrm{mm}^{2}$ ). El comportamiento de las probetas de Eucalyptus grandis preparadas en clima normalizado fue similar al de otras de la misma especie elaboradas a superior temperatura $\left(23^{\circ} \mathrm{C}\right)$ y humedad relativa ambiente $(84 \%)$.

\section{REFERENCIAS}

AMERICAN SOCIETY FOR TESTING AND MATERIALS. ASTM D 3737-99: standard practice for establishing stresses for structural glued laminated timber (Glulam). West Conshohocken, 1999.

COLLING, F. Glued laminated timber: production and strength classes. In: TIMBER Engineering step 1. The Netherlands: Centrum Hout,, 1995a. p. A8/1-A8/8.

COLLING, F. Brettschichtholz unter Biegebeanspruchung. In: INFORMATIONSDIENST Holz, step 3. Düsseldorf : Fachverlag Holz, 1995b. p. 5/1-5/18. 
COLLING, F.; EHLBECK, J. Tragfähigkeit von Keilzinkenverbindungen im Holzleimbau. Bauen mit Holz, Karlsruhe, v. 7, n. 92, p. 586-593, 1992.

EUROPÄISCHES KOMITEE FÜR NORMUNG. PR EN 301: Klebstoffe für tragende Holzbauteile, Phenoplaste und Aminoplaste, Klassifizierung und Leistungsandorderungen. Berlin: Beuth, 2001.

EUROPÄISCHES KOMITEE FÜR NORMUNG. DIN EN 302-1: Klebstoffe für tragende Holzbauteile, Prüfverfahren, Teil 1: Bestimmung der Längszugscherfestigkeit. Berlin: Beuth, 2004.

EUROPÄISCHES KOMITEE FÜR NORMUNG. EN 338: Bauholz für tragende Zwecke, Festigkeitsklassen. Berlin: Beuth, 2003.

EUROPÄISCHES KOMITEE FÜR NORMUNG. EN 386: Brettschichtholz, Leistungs- und Mindestanforderungen an die Herstellung. Berlin: Beuth, 1996.

EUROPÄISCHES KOMITEE FÜR NORMUNG. DIN EN 1194: Holzbauwerke, Brettschichtholz, Festigkeitsklassen und Bestimmung charakteristischer Werte. Berlin: Beuth, 1999.

GLOS, P.; LEDERER, B. Sortierung von Buchen- und Eichenschnittholz nach der Tragfähigkeit und Bestimmung der zugehörigen Festigkeits-und Steifigkeitskennwerte. Bericht Nr. 98508. München: Institut für Holzforschung, 2000.

HOFFMEYER, P. Wood as a building material. In: TIMBER Engineering step 1. The Netherlands: Centrum Hout, 1995. p. A4/1-A4/21.

INSTITUTO ARGENTINO DE NORMALIZACIÓN. IRAM 45054-1: Adhesivos para estructuras de madera bajo carga, Métodos de ensayo, Parte 1: Determinación de la resistencia de la unión a la cizalladura por tracción longitudinal. Buenos Aires, 2002a.

INSTITUTO ARGENTINO DE NORMALIZACIÓN. IRAM 45055: Adhesivos para estructuras de madera bajo carga, Adhesivo de policondensación de tipos fenólicos, aminoplásticos y de otros tipos, Clasificación y requisitos de comportamiento. Buenos Aires, 2002b.

INSTITUTO NACIONAL DE TECNOLOGÍA AGROPECUARIA - INTA. Manual para Productores de Eucaliptos de la Mesopotamia Argentina. Concordia: 1995.

INTERNATIONAL ORGANIZATION FOR STANDARDIZATION. ISO 3130-1975 (E): Wood Determination of moisture content for physical and mechanical tests. Geneva, 1975a.

INTERNATIONAL ORGANIZATION FOR STANDARDIZATION. ISO 3131-1975 (E): Wood Determination of density for physical and mechanical tests. Geneva, $1975 \mathrm{~b}$.

PITER, J. C. Mejora de la calidad de las vigas laminadas encoladas para uso estructural en construcciones: experiencia de innovación y transferencia entre el sector científico-tecnológico y el empresario en Argentina. SEMINARIO Y MINI FORO IBEROAMERICANO DE TECNOLOGÍA DE MATERIALES, 2005, Habana. [Annales...]. Madrid: CYTED, 2005.

PITER, J. C.; ZERBINO R. L.; BLA $\beta$ H. J. Visual strength grading of Argentinean Eucalyptus grandis. Strength, stiffness and density profiles and corresponding limits for the main grading parameters. Holz als Roh und Werkstoff, Berlin, v. 62, p. 1-8. 2004.

RADOVÍC, B. Leime und Leimverbindungen. In: INFORMATIONSDIENST Holz, step 3. Düsseldorf: Fachverlag Holz, 1995. p. 6/1-6/21.

RAKNES E.. Adhesives. In: TIMBER Engineering step 1. The Netherlands: Centrum Hout, 1995. p. A12/1-A12/8. 